\title{
Educação permanente em equipe multidisciplinar de um programa gerontológico: concepções, desafios e possibilidades
}

\section{Permanent education in multidisciplinary team from a geriatric program: concepts, challenges and possibilities}

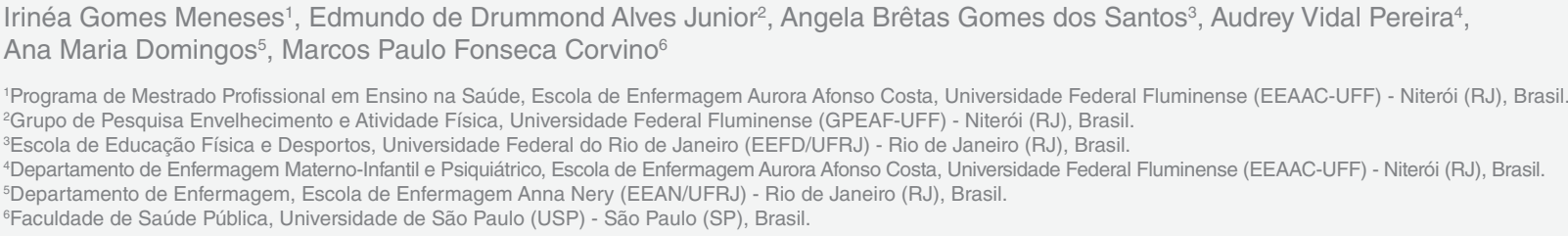

DOI: https://dx.doi.org/10.7322/abcshs.v44i1.1257

\section{RESUMO}

Introdução: $O$ aumento progressivo da população idosa e das enfermidades crônicas torna cada vez mais prioritário a educação permanente dos profissionais de saúde visando o atendimento das necessidades dos idosos. Objetivo: Conhecer as concepções da equipe multiprofissional e de gestores sobre Educação Permanente de um Programa de Assistência Integral à Pessoa Idosa. Métodos: Trata-se de um estudo descritivo com abordagem qualitativa. $O$ cenário foi um Programa Gerontológico desenvolvido em um Instituto de Atenção à Saúde de uma Universidade Pública, no município do Rio de Janeiro. Os participantes foram 10 profissionais de saúde incluindo os gestores do referido programa. Foi utilizado como técnica de coleta de dados o grupo focal, cujas reflexões ocorreram a partir de análise de conteúdo na modalidade temática. Resultados: A pesquisa permitiu a construção de duas categorias: 1) Educação Permanente como movimento de atualização profissional e 2) Educação Permanente: aproximando o interesse pessoal, iniciativa institucional e necessidades dos usuários. Pode-se observar que a educação permanente ocorre a partir da identificação de problemas cotidianos e necessidades dos serviços e usuários do sistema de saúde, que se envolve com a responsabilidade de melhorar as condições de saúde da população de idosos atendida. Conclusão: O estudo contribuiu para uma reflexão que se traduza na organização do processo de trabalho e na busca pelo aprimoramento da prática assistencial qualificada e resolutiva, com vistas à operacionalização futura de processos de Educação Permanente.

Palavras-chave: educação em saúde; educação continuada; pessoal de saúde; planos e programas de saúde; envelhecimento.

\begin{abstract}
Introduction: The progressive increase of the elderly population and chronic diseases turns the permanent education of health professionals more and more urgent in order to meet the needs of the elderly. Objective: To know the conceptions of the multiprofessional team and of managers on Permanent Education of a Program of Integral Assistance to the Elderly. Methods: This is a descriptive study with a qualitative approach. The scenario was a Geriatric Program offered in a Health Care Institute of a Public University in the city of Rio de Janeiro. The participants were 10 health professionals including the managers of the program. Focal group was used as data collection technique, whose reflections occurred from content analysis in the thematic modality. Results: The research allowed the construction of two categories: 1) Permanent Education as a professional updating movement and 2) Permanent Education: approaching personal interest, institutional initiative and users needs. It can be observed that permanent education occurs from the identification of daily problems and the needs of the services and the users of the health system, being involved with the responsibility of improving the health conditions of the elderly population served. Conclusion: The study contributed to a reflection that translates into the organization of the work process and the search for the improvement of the qualified and resolutive assistance practice, with a view to the future operationalization of Permanent Education processes.
\end{abstract}

Keywords: health education; education, continuing; health personnel; health programs and plans; aging.

Recebido em: 26/07/2017

Revisado em: 08/06/2018

Aprovado em: 31/10/2018

Autor para correspondência: Irinéa Gomes Meneses - Escola de Enfermagem Aurora Afonso Costa, Universidade Federal Fluminense - Avenida Roma 176 Casa 01 - CEP: 21041-060 - Bonsucesso (RJ), Brasil - E-mail: irineameneses@bol.com.br

Conflito de interesses: nada a declarar. 


\section{INTRODUÇÃO}

De acordo com a Portaria GM no 1.996 GM/MS, de 20 de agosto de 2007 a educação permanente é o conceito pedagógico que é capaz de intermediar ações de ensino, serviços, gestão e desenvolvimento de instituições. Esta portaria instituiu a Política Nacional de Educação Permanente em Saúde ${ }^{1,2}$ como uma estratégia do SUS, dispondo sobre a formação e o desenvolvimento de trabalhadores do setor saúde.

No sentido de ocorrer mudanças relacionadas às formações dos profissionais no âmbito da saúde, o Ministério da Saúde propôs a educação permanente como "estratégia de transformação das práticas de formação, atenção, gestão, formulação de políticas, participação popular e do controle social e da saúde"3. Assim, foi lançada a Política de formação e desenvolvimento para o SUS ${ }^{4}$, baseada na proposta de educação permanente, com a finalidade de normatizar a organização dos processos de gestão da educação em saúde nos diferentes campos.

Neste sentido, a Educação Permanente em Saúde (EPS) é considerada no "âmbito da saúde uma categoria relacionada ao crescimento de uma escuta do cuidado e do tratamento, que na intervenção ou na interferência do indivíduo e de um grupo, há um cultivo de aprendizado relativo"s.

Sendo assim, a problematização na EPS contribui para a construção do conhecimento na área da saúde por meio de atos que são produzidos no cotidiano, e a partir desta observação, é possível formular processos educativos capazes de transformar as práticas em saúde ${ }^{6}$. A EPS tem "relação com o exercício cotidiano do processo de trabalho; com a valorização da ocupação profissional como fonte de conhecimento, bem como na articulação entre atenção à saúde, gestão e controle social, centrada na multiprofissionalidade e interdisciplinaridade, com estratégias de ensino contextualizadas e participativas"7. Assim, EPS é um grande desafio que integra trabalho, educação e saúde, contemplando experiências pessoais e conhecimentos, agregando nesse processo, usuários, serviços de saúde, gestão e instituições de ensino.

Deste modo, o trabalho de equipe multiprofissional no campo da gerontologia brasileira também necessita operacionalizar estratégias a fim de assegurar políticas públicas de educação permanente, que efetivem uma atenção à saúde de qualidade, visto que o Brasil está passando por um período de envelhecimento populacional. Segundo a pesquisa nacional por amostra de domicílios o crescimento esperado da proporção de pessoas de 60 anos ou mais de idade na população brasileira, a partir de 2010 tem se aproximado ao projetado nos países desenvolvidos, cuja estimativa seria em 2070 acima de $35 \%{ }^{1}$.

Neste contexto, com o elevado crescimento da população idosa destaca-se a importância do envolvimento dos profissionais de saúde em ações de educação permanente junto aos idosos, sobretudo por meio de atividades específicas relacionadas a programas de ensino, pesquisa e extensão referentes ao envelhecimento. Esta estratégia se configura como um modelo diferenciado de atenção básica a saúde das pessoas idosas, visto que tem a intenção de provocar dinamismo no trabalho interdisciplinar, além de suscitar a participação do próprio grupo de idosos.

No programa são realizadas atividades pela equipe multiprofissional que contribuem para a formação técnica-científica e para atenção integral à saúde dos usuários idosos, cujo foco está relacionado ao acolhimento das demandas biopsicossociais desta população atendida.

Desta forma, têm-se como objetivo conhecer as concepções da equipe multiprofissional e de gestores sobre Educação Permanente, de um Programa de Assistência Integral à Pessoa Idosa.

\section{MÉTODOS}

Trata-se de um estudo descritivo com abordagem qualitativa, realizado no período de agosto a outubro de 2017. Foi utilizado referencial conceitual de educação permanente e modalidades assistências no âmbito da gerontologia.

A pesquisa teve como cenário o Programa de Assistência Integral à Pessoa Idosa (PAIPI) no Instituto de Atenção à Saúde São Francisco de Assis, na Universidade Federal do Rio de Janeiro. A opção por este Programa ocorreu mediante a possibilidade de registrar ações de ensino, pesquisa e extensão no âmbito da referida Universidade.

As atividades oferecidas pelo Programa de Assistência Integral à Pessoa Idosa perpassam ações informativas, reflexivas, sociais, educativas e assistenciais ambulatoriais.

Os participantes deste estudo foram 10 (dez) profissionais da equipe multiprofissional, incluindo os gestores. Neste sentido, os critérios para participação na pesquisa foram: 1) critérios de inclusão: profissionais da equipe multiprofissional ou gestores do programa gerontológico que se encontrava em pleno exercício de suas funções há mais de um ano e possuidores de vínculo empregatício efetivo (servidor público); 2) critérios de exclusão: profissionais licenciados ou em período de férias.

Com vistas à obtenção das informações utilizou-se o grupo focal. A sua realização prevê a produção de dados mediante discussões planejadas em grupo, especialmente na produção de dados qualitativos, pois os participantes interagem e expõem suas percepções, representações, valores e crenças sobre um dado fenômeno ${ }^{8}$.

Para orientar o processo de investigação foi utilizado um guia de perguntas, como técnica de produção de dados, sendo a duração das respectivas sessões foi de aproximadamente duas horas.

A efetivação do grupo focal ocorreu com um pequeno número de participantes permitindo participação eficaz e discussão coerente dos temas estudados ${ }^{9}$, que foi dividido em dois, sendo constituído por cinco pessoas em cada sessão, totalizando dez participantes na pesquisa, e pela pesquisadora que atuou como moderadora. 
Após aprovação no Comitê de Ética em Pesquisa (Parecer de aprovação $\mathrm{n}^{\circ}$ 1.920.309) e respectiva autorização da instituição, iniciou-se a primeira sessão.

A Primeira Sessão com um breve relato sobre a pesquisa com a explanação dos objetivos propostos. Foi entregue aos participantes o Termo de Consentimento Livre e Esclarecido (TCLE), para a leitura e assinatura de concordância, em duas vias, sendo uma delas entregue à pesquisadora. Também foram esclarecidos quanto à dinâmica do grupo focal e a importância da participação de cada integrante do grupo. Após este momento, deu-se início a primeira sessão do grupo focal com uma dinâmica de grupo, intitulada a Dinâmica do Sonhar acordado ${ }^{10}$, que durou aproximadamente 20 minutos. Com o som ligado em uma música para relaxamento, a pesquisadora orientou, aos participantes da pesquisa que fechassem seus olhos e seguissem as orientações conforme as questões abaixo. Esta sessão foi realizada com os seguintes temas: 1) O que é Educação Permanente? 2) Do ponto de vista pessoal e profissional, a participação em um Programa de Educação Permanente pode influenciar no seu processo de trabalho?

A segunda sessão compreendeu a continuidade da discussão dialógica em torno das seguintes questões: 1) O que dificulta do ponto de vista pessoal, profissional, e institucional a participação em um Programa de Educação Permanente? 2) Que conteúdos, práticas gerontológicas e ações devem fazer parte de um Programa de Educação Permanente no PAIPI?

Para análise do material coletado no grupo focal foram utilizados os procedimentos preconizados por Bardin ${ }^{11}$ no que se refere à técnica de análise de conteúdo na modalidade temática.

\section{RESULTADOS E DISCUSSÃO}

A pesquisa permitiu a construção de duas categorias originadas a partir das falas dos entrevistados, as quais são: 1) Educação Permanente, como movimento de atualização profissional e 2) Educação Permanente: aproximando o interesse pessoal, iniciativa institucional e necessidades dos usuários.

\section{Categoria 1) Educação Permanente como movimento de atualização profissional}

Nesta categoria foram abordadas temáticas relacionadas ao processo de educação permanente como atualização contínua individual e o aprendizado cotidiano e processual como modo de enfrentar as mudanças que ocorrem no dia a dia dos serviços de saúde. Os entrevistados apontaram para a necessidade de atualização constante e busca de aprimoramento profissional, por meio de participações em congressos e seminários, por exemplo, direcionado ao seu próprio desempenho com vistas às transformações ocorridas no processo de trabalho. Isso pode ser observado no depoimento a seguir:
É a continuidade de aperfeiçoamento profissional de profissionais em modalidades de atualização presencial/distancia, de grupos de estudo direcionado, quando buscamos fazer uma especialização teórico e pratica em determinada área de interesse, participar de congresso nacionais e internacionais, fóruns de debates, cursar o mestrado acadêmico/profissional, o doutoramento e pós-doutoramento. Compreende as várias possibilidades de agregar conhecimento e experiências de um profissional [...]. No meu caso o campo da saúde, onde atuo (envelhecimento humano e centro de convivência para pessoas idosas), se torna vital o aperfeiçoamento e capacitação para conseguir interagir com as demandas constantes e diversificadas entre os indivíduos [...] (Participante 9).

A necessidade de uma educação a partir da demanda/realidade cotidiana, se torna presente no estudo do Programa Nacional de Educação Permanente de Saúde (PNEPS), destacando um significado de política pública em ampliação como um aprendizado no trabalho para a constituição do Sistema Único de Saúde ${ }^{12}$.

Neste contexto, a Educação Permanente foi vista pelos participantes como uma construção de um processo de trabalho, que caracteriza mudanças contínuas a partir de um aprendizado cotidiano, sendo a atualização contínua uma forma associada ao processo de formação cuja ferramenta se torna necessária para o processo de trabalho ${ }^{7}$. Este fato pode ser identificado através do depoimento a seguir:

[...] É um aprendizado constante para lidar com os desafios do dia a dia, que são variáveis que mudam ao longo do tempo, nada é estático, o que eu aprendi não vou utilizar sempre da mesma forma, vai sofrer uma série de transformações de acordo com as mudanças do meio [...] a educação permanente (EP) é necessário para evolução de qualquer processo de trabalho [...] (Participante 5).

É importante ressaltar que a Educação Permanente é construída a partir do processo de trabalho cotidiano e se expressa como uma categoria de ações educativas, que visa o desenvolvimento de atividades direcionadas ao processo de ensino-aprendizado relacionado às demandas do trabalho ${ }^{13}$. Desta forma, a EPS contribui para transformar ações individuais a partir da identificação da realidade, como pode ser visto a partir do seguinte depoimento:

[...] Você se manter atualizado, ainda mais que hoje a gente tem uma ferramenta muito poderosa, que é a internet. [...] É o tempo todo ficar pesquisando e lendo, então a educação permanente, é isso, você está sempre indagando, sobre a suas atividades, o seu serviço, sobre a sua formação, porque as coisas estão muito rápidas, com essa ferramenta, antes eu tinha que procurar periódicos, hoje, os periódicos estão online. [...] (Participante 1). 
É relevante a reflexão que no processo de atualização é importante que o profissional pense na qualidade do conhecimento que deve ser apreendido e como fazer para que as pessoas adquiram este conhecimento ${ }^{2}$, principalmente os que estão inseridos no meio digital.

Diante das informações dos entrevistados, pode-se perceber que há uma busca pela atualização de forma que qualifique a própria assistência profissional, viabilizando a identificação de ações para lidar com os desafios que surgem no dia a dia.

[...] A Educação Permanente para mim é, a permanência da necessidade de atualização. Você tem que estar sempre se atualizando para estar levando o melhor, o questionamento vem a partir do momento em que você leva informação, cria um círculo de perguntas em cima daquela informação que você está levantando, está sempre buscando, se atualizando [...] (Participante 2).

Como se pode observar, a necessidade de operacionalizar ações de educação permanente relacionadas à população em processo de envelhecimento vai para além de serviços específicos como no caso deste estudo; vide recomendações de sustentar processos de educação permanente para profissionais já inseridos nos serviços como em unidades de saúde da família ${ }^{14}$.

A busca pela atualização voltada para uma população específica qualifica o cuidado, tornando-o mais eficaz e mais responsivo às reais necessidades dos usuários dos serviços de saúde ${ }^{9}$; podendo as demandas que emergem no cotidiano de trabalho, serem percebidas por meio de um olhar mais aguçado do profissional ${ }^{15}$. Mesmo que seja tímido o entendimento do grupo pesquisado de que educação permanente deva prioritariamente desenvolver-se num processo de identificação de problemas cotidianos, existem diretrizes a serem alcançadas apontando que a Educação Permanente seja realizada a partir do reconhecimento profissional do contexto de trabalho no qual se encontra inserido ${ }^{16}$. Esta característica pode ser identificada a partir da fala a seguir:

[...] Entendo a educação como um processo que a gente envolve uma aprendizagem que pode ser feita de várias maneiras dentro de um ambiente de trabalho. [...] A educação permanente em qualquer serviço é necessária, ela pode se fazer a partir primeiro, de uma percepção do próprio profissional. [...] Se você está percebendo como o contexto que te rodeia, a clientela que está ali dependendo dos seus cuidados, como ela também, se coloca naquele ambiente de trabalho, quais são as demandas que aparece no dia a dia, como a equipe que te rodeia também trabalha, tudo isso, se você está atenta, você vai se renovando [...] A Educação Permanente, depende muito também do profissional em si, ela, é necessária sim, o tempo todo, ela não pode ser um programa instituído que funciona uma vez a cada três anos, uma vez por ano, não, ela tem que ser diária, um processo dinâmico que está ali caminhando de acordo com o seu fazer assistencial [...] Então envolve três questões [...] o usuário, próprio profissional e a instituição (Participante seis).

Deve estar presente na vida dos profissionais em movimentos contínuos, sendo desenvolvida prioritariamente no sentido de 'oficinas interativas' ou 'reuniões educativas' do que em formatos de exposições ou capacitações tradicionais ${ }^{17}$.

Devido ao modo de trabalhar com condições de complexidades, direcionando a possibilidade de mudança constante no processo de trabalho ${ }^{18}$. A educação a partir da demanda da realidade cotidiana é um fator importante para um cuidado direcionado às pessoas inseridas em uma determinada realidade sócio institucional, que vai além de capacitações pontuais em formatos de cursos ou participações em congressos. Esta característica pode ser observada através do depoimento a seguir:

[...] É a gente ter consciência de que não sabemos tudo, que mesmo estando formados há 10, 20, 30 anos, o mundo e a assistência em saúde, estão em constante movimento, então a cada dia que passa a gente precisa estar se preparando para atender a demanda que chega para a gente. Então educação permanente, não é só fazer um curso lato Sensu ou Stricto $S e n s u$, às vezes é buscar pequenos cursos, artigos, revistas, as informações, nos noticiários, nos documentários e nos filmes, onde você pode buscar informação, para você se atualizar, e você responder as demandas das pessoas que chegam até você [...] (Participante 3).

Assim, os profissionais de saúde envolvidos devem transformar o grupo de trabalho em uma equipe que consiga de forma organizada se desenvolver e atuar junto aos usuários e sociedade a fim de resolver as inúmeras situações ou problemas que aparecem no dia a dia dos serviços de saúde ${ }^{16}$.

\section{Categoria 2) Educação Permanente: aproximando $o$ interesse pessoal, iniciativa institucional e necessidades dos usuários.}

Em alguma medida existe uma concepção por parte dos profissionais participantes deste estudo de que educação permanente ainda se apresenta de modo polarizado com a ideia de educação continuada em serviço. As falas dos participantes apontam para uma conotação de educação relacionada à capacitação profissional, surgindo uma possibilidade de espera por uma eventual organização de cursos institucionais. Assim, denota ideia de processo de qualificação, curso associado ao plano de cargos e incentivo acadêmico.

A busca pela capacitação dos profissionais por meio de Congressos e Seminários está presente em falas do grupo, que 
pode ser exemplificado quando o profissional refere que educação permanente é: “... buscar pequenos cursos, artigos, revistas...” (Participante 3).

A capacitação técnica das profissões, muitas vezes, é realizada de forma desintegrada da gerência com os profissionais que estão na área assistencial.

Desta forma, dificultando o acesso aos novos conhecimentos a esses profissionais ${ }^{19}$. Além do que, ainda encontram-se inúmeras dificuldades para assegurar um processo de educação permanente no âmbito da atenção à saúde de idosos. Vide que a formação especializada e contínua em geriatria ainda é desproporcional à crescente demanda da população de idosos ${ }^{20}$.

A espera pelos incentivos institucionais dos participantes em muitas das vezes fica relacionada à falta de investimento na qualificação do funcionário e incentivo institucional, como pode ser visto a seguir: “... a gente não tem uma educação permanente que vem de fora para nós, de alguém que nos oferece, da instituição que nos oferece..." (Participante 6).

No entanto, o investimento na qualificação profissional valoriza o profissional, possibilitando que o seu cuidado possa ser mais qualificado e direcionado ao indivíduo ${ }^{21}$. Deste modo, pode-se destacar que a realização do investimento acadêmico por parte da instituição também pode ser caracterizado positivamente como processo de educação permanente. Vide o incentivo para aperfeiçoamento profissional através da realização de cursos, como no caso dos planos de cargos e salários. Tal circunstancia pode ser observada quando uma profissional diz que “... pelo menos na faculdade tem um incentivo de qualificação para aumentar o meu salário..." (Participante 8 ).

É imprescindível a modificação do cenário de aperfeiçoamento profissional por parte daqueles que discutem as políticas ${ }^{2}$, pois é fundamental que o gestor do serviço elabore propostas aos seus funcionários, que sejam benéficas e possam trazer satisfação e qualificação aos mesmos no ambiente de trabalho. Pois, a falta de investimentos relacionados ao crescimento profissional pode causar desmotivação para o envolvimento em capacitações ${ }^{22}$. Os participantes mencionaram a necessidade de buscar informação e maneiras de investir na apropriação do conhecimento, de modo a incrementar sua atuação profissional tendo em vista as necessidades cotidianas no contexto do trabalho em saúde. Isso pode ser observado nos depoimentos a seguir:

[...] Possibilita sua atualização, você está sempre por dentro do que está acontecendo, uma atualização, sobre os mais variados temas, para que a gente tenha aprendizado. [...] Eu acho que facilita dessa maneira, de que você se mantem atualizado a todo tempo (Participante 2).

Os entrevistados abaixo demonstram em seus relatos que ao buscarem capacitação, se qualificarem, terem acesso a novas tecnologias, novos métodos e novas informações, as mesmas se tornam profissionais melhores e têm uma melhor resposta na assistência.

[...] Ter acesso, buscar todo o processo de atualização de capacitação permanente; a educação permanente qualifica a minha prática. [...] Buscar capacitação permanente (Participante 3).

A educação permanente no trabalho se transforma em uma ferramenta de mudança institucional, promovendo a compreensão integral argumentado pelo grupo, fundamentando-se na problemática do serviço, conduzindo à autocrítica e a reflexão ${ }^{23}$ que por sua vez retorna como perspectiva de mudança para a prática cotidiana dos serviços de saúde. Pois o conhecimento tem sido utilizado como recurso no planejamento de ações inteligentes e eficazes nas organizações sendo responsável para projetar resultados almejados, além de expandir padrões de pensamentos de interesses coletivos ${ }^{24}$.

[...] É lógico. Se a instituição me oferece um Curso de Cognição para idosos para você fazer, ótimo. Eu não tenho, eu tenho que correr atrás. Tenho que fazer, tenho que buscar, aprender, que ler sobre o assunto, escrever. A coordenação acadêmica, quer que eu escreva um Projeto da Oficina de Memória. Escrevi aqui no PAIPI, mas ela quer que eu faça um artigo, acho que a porta é primeiro, essa percepção que você (Pesquisadora) falou, logo no início. A princípio tem que perceber o que a instituição vai precisando, o que eu como profissional estou precisando, o que os idosos estão precisando. É um triangulo, das três necessidades (Participante 6).

Assim, a EPS conta ainda com estratégias de governabilidades diferenciadas e requer incentivos institucionais que vão para além de iniciativas individuais, pois é um processo que convive com dificuldades que são vivenciadas no cotidiano dos serviços, gestores, usuários e pelo trabalho da equipe de saúde; como pode ser observado a seguir:

[...] é uma política que dá materialidade ao encontro entre educação e a saúde. Isso se dá no cotidiano do trabalho, pela problematização das dificuldades vivenciadas na realidade, e perpassa pelo reconhecimento do protagonismo do trabalhador e do usuário, e pela necessidade de se promover mudanças no processo de trabalho no campo da saúde (Participante 10).

A operacionalização de uma rede entre o usuário, profissional e instituição fará com que a atualização seja contínua no processo de trabalho. A transformação do cotidiano deve ser levada em conta no processo de cuidado, para que o serviço seja realizado 
com melhor qualidade envolvendo gestão, assistência e participação. A educação permanente é uma alternativa para os profissionais da área de saúde sejam capazes de analisar as mudanças ocorridas no cotidiano e avaliar o seu processo de trabalho como um todo. Este fato é observado no depoimento a seguir:

[...] É um aprendizado constante para lidar com os desafios do dia a dia, que são variáveis que mudam ao longo do tempo, nada é estático, o que eu aprendi não vou utilizar sempre da mesma forma, vai sofrer uma série de transformações de acordo com as mudanças do meio [...] a educação permanente (EP) é necessário para evolução de qualquer processo de trabalho (Participante 5).

Nesse contexto, torna-se necessária uma interferência concomitante voltada às necessidades dos trabalhadores da saúde para operar nesse cenário específico, contemplando um aperfeiçoamento técnico científico e atualização permanente. "Artigos de todas as décadas mostram a necessidade de investir no trabalhador para a construção de uma assistência humana considerando, inclusive, as condições adversas de trabalho...”25.

As instituições necessitam reconhecer o avanço e as transformações expressivas das competências e dos aprendizados da equipe profissional em todos os níveis. Pode-se perceber que é de grande importância que a instituição dê oportunidades aos profissionais, estimulando-os para que tenham um desenvolvimento profissional.

No meu caso sendo profissional estatutário ligado diretamente a Universidade Federal que colabora com a minha capacitação ao longo de treze anos. O Plano de Cargo e Salários assegura esse direito a nós servidores (ter a liberação parcial ou total para realizar essas atividades). A instituição onde sou locada também não apresenta obstáculos para os profissionais...
Minha experiência pessoal tanto para me especializar, quanto para fazer o mestrado ou sair para congressos nacionais e internacionais foi boa (Participante 9).

Para tal, a progressão por capacitação profissional decorrente da obtenção pelo servidor de certificação em Programa de Capacitação, compatível com o cargo ocupado, o ambiente organizacional e a carga horária mínima exigida promove incentivo à qualificação profissional ${ }^{26}$.

Em suma, o processo de trabalho necessita ser orientado por meio do envolvimento e trabalho em equipes, cuja ideia de "equipe agrupamento" deve se contrapor à noção de "equipe integração" onde "ocorre à articulação das ações e a interação dos agentes"

Assim, a PNEP recomenda que as técnicas da formação dos profissionais de saúde, contemplem a precisão de formação e que o progresso desses trabalhadores seja orientado às necessidades de atualização para a saúde da população ${ }^{2}$, pois é a partir da identificação de problemas cotidianos e necessidades dos serviços e usuários do sistema de saúde, que se confirma a responsabilidade de melhorar as condições de saúde da população de idosos atendida.

Em que pese às contribuições do presente este estudo, vale destacar que apresenta limitações relacionadas com um número limitado de profissionais participantes sendo realizado em apenas um único local.

Em conclusão, o estudo colabora ao cenário nacional sobre EP à uma equipe com foco na atenção à pessoa idosa e envelhecimento e contribui para uma reflexão que se traduza na organização do processo de trabalho e na busca pelo aprimoramento da prática assistencial qualificada e resolutiva. Através dos resultados desse trabalho podem-se ter subsídios para planejar normas de melhoria em um método educativo em um Programa Gerontológico, com vistas à operacionalização futura de processos de Educação Permanente.

\section{REFERÊNCIAS}

1. Brasil. Ministério da Saúde. Secretaria de Gestão do Trabalho e da Educação na Saúde. Departamento de Gestão da Educação em Saúde. Política nacional de educação permanente em saúde. Brasília: Ministério da Saúde, 2009.

2. Brasil. Ministério da Saúde. Secretaria de Gestão do Trabalho e da Educação na Saúde. Departamento de Gestão da Educação em Saúde. A educação permanente entra na roda. Pólos de educação permanente em saúde. Conceitos e caminhos a percorrer. Brasília: Ministério da Saúde, 2005.

3. Brasil. Ministério da Saúde. Portaria GM/MS n 198, de 13 de fevereiro de 2004. Institui a Política Nacional de Educação Permanente em Saúde como estratégia do Sistema Único de Saúde para a formação e o desenvolvimento de trabalhadores para o setor e dá outras providências. Disponível em: https://www. nescon.medicina.ufmg.br/biblioteca/imagem/1832.pdf. Acesso em: 8 jun. 2018.
4. Ceccim RB. Educação permanente em saúde: desafio ambicioso e necessário. Interface: Comunic Saúde Educ. 2005;9(16):161-77.

5. Massaroli A, Saupe R. Distinção Conceitual: educação permanente e educação continuada no processo de trabalho em Saúde. Disponível em: http://www1.saude.rs.gov.br/ dados/1311947098405educa\%E7\%E30\%20continuada\%20 e\%20permanente.pdf. Acesso em: 30 abr. 2018.

6. Peduzzi M, Guerra DAD, Braga CP, Lucena FS, Silva JAM Atividades educativas de trabalhadores na atenção primária: concepções de educação permanente e de educação continuada em saúde presentes no cotidiano de Unidades Básicas de Saúde em São Paulo. Interface. 2009;13(30):121-34.

http://dx.doi.org/10.1590/S1414-32832009000300011

7. Instituto Brasileiro de Geografia e Estatística (IBGE). Pesquisa nacional por amostra de domicílios: síntese de indicadores 2015. Rio de Janeiro: IBGE, 2016; p.105. 
8. Backes DS, Colomé JS, Erdmann RH, Lunardi VL. Grupo focal como técnica de coleta e análise de dados em pesquisas qualitativas. Mundo Saúde. 2001;35(4):438-42.

9. Pizzol SJS. Combinação de grupos focais e análise discriminante: um método para tipificação de sistemas de produção agropecuária. Rev Econ Sociol Rural. 2004;42(3):451-68. http://dx.doi.org/10.1590/S0103-20032004000300003

10. Centro de Estudos Espíritas Allan Kardec (CEEAK). Dinâmicas de integração e aquecimento. Sonhar acordado (relaxamento). Disponível em: https://www.dij.ceeak.ch/dinmicas/dinmicas de integraco e aquecimento/sonhar acordado relaxamento/index. html. Acesso em: 30 abr. 2018.

11. Bardin L. Análise de conteúdo. São Paulo: Edições 70, 2011; p.229.

12. Brasil. Ministério da Saúde. Portaria GM/MS $n^{\circ} 1.996$, de 20 de agosto de 2007. Dispõe sobre as diretrizes para a implementação da Política Nacional de Educação Permanente em Saúde. Disponível em: http://bvsms.saude.gov.br/bvs/ saudelegis/gm/2007/prt1996 2008 2007.html. Acesso em 30 abr. 2018.

13. Motta JIJ, Buss $P$, Nunes TCM. Educação Permanente em Saúde. Rev Olho Mágico. 2002;9(1):67-78.

14. Silva KM, Santos SMA. A práxis do enfermeiro da estratégia de saúde da família e o cuidado ao idoso. Texto Contexto Enferm. 2015;24(1):105-11.

http://dx.doi.org/10.1590/0104-07072015000680013

15. Queiroz DL, Souza JC. Qualidade de vida e capacidade para o trabalho de profissionais de enfermagem. Psicol Inf. 2012;16(16):103-26

16. Ceccim RB, Ferla AA. Educação Permanente em Saúde. In: Fundação Oswaldo Cruz. Escola Politécnica de Saúde Joaquim Venâncio. Dicionário da Educação Profissional em Saúde. Rio de Janeiro: FIOCRUZ; 2006; p.107-12

17. Forsetlund L, Biørndal A, Rashidian A, Jamtvedt G, O'Brien MA, Wolf $F$, et al. Continuing education meetings and workshops: effects on professional practice and health care outcomes. Cochrane Database Syst Rev. 2015;15(2):CD003030. http://dx.doi.org/10.1002/14651858.CD003030.pub2
18. Fernandez A, Schillinger D, Grumbach K, Rosenthal A, Stewart AL, Wang $F$, et al. Physician language ability and cultural competence. An exploratory study of communication with spanish-speaking patients. J Gen Intern Med. 2004;19(2):167-74 https://dx.doi.org/10.1111/j.1525-1497.2004.30266.x

19. Vincent SP. Educação permanente: componente estratégico para a implementação da política nacional de atenção oncológica. Rev Bras Cancerol. 2007;53(1):79-85.

20. Bardach SH, Rowles GD. Geriatric education in the health professions: are we making progress? Gerontologist 2012;52(5):607-18. https://dx.doi.org/10.1093/geront/gns006

21. Kraemer FB, Aguiar OB. Gestão de competências e qualificação profissional no segmento da alimentação coletiva. Rev Nutr. 2009;22(5):609-19.

http://dx.doi.org/10.1590/S1415-52732009000500002

22. Oliveira MS, Moreira MLS. Motivação e clima organizacional no ambiente de trabalho. Monografia (Pós-Graduação em Gestão de Recursos Humanos) - Faculdade Cândido Mendes. Rio de Janeiro: 2010

23. Daviani MC. Enfoques, problemas e perspectivas na educação permanente dos recursos humanos de saúde. In: Brasil. Ministério da Saúde. Secretaria de Gestão do Trabalho e da Educação na Saúde. Departamento de Gestão da Educação em Saúde. Política Nacional de Educação Permanente em Saúde. Brasília: 2009; p.39-63.

24. Rocha ESB, Nagliate P, Furlan CEB, Rocha Jr K, Trevisan MA, Mendes IAC. Gestão do conhecimento na saúde: revisão sistemática de literatura. Rev Latino-Am Enfermagem. 2012;20(2):392-400. http://dx.doi.org/10.1590/S0104-11692012000200024

25. Casate JC, Corrêa AK. Humanização do atendimento em saúde: conhecimento veiculado na literatura brasileira de enfermagem. Rev Latino-Am Enfermagem. 2005;13(1):105-11. http://dx.doi.org/10.1590/S0104-11692005000100017

26. Brasil. Presidência da República. Lei $n^{\circ} 11.784$, de 22 de setembro de 2008. Dispõe sobre a reestruturação do plano geral de cargos do poder executivo. Disponível em: http://www.planalto.gov.br/cciVil 03/ Ato2007-2010/2008/Lei/L11784.htm. Acesso em: 30 abr. 2018. 